\title{
Los grupos de investigación en la UNAH: unidades fundamentales para la producción de conocimiento y formación de relevo científico
}

Iving Zelaya ${ }^{1}$

En este artículo se expone el estado actual de los grupos de investigación que forman parte del Sistema de Investigación Científica y Tecnológica de la UNAH, sus aportes a la institucionalización de la actividad científica y los principales avances que se han mostrado en el marco de una estrategia orientada hacia el fomento de estas unidades funcionales.

La organización de la estructura de investigación en las instituciones de educación superior es un tema de especial importancia a la luz de los cambios que se han venido produciendo en los mecanismos de producción del conocimiento y los reclamos de eficiencia y eficacia en los procesos de investigación universitarios (Rey Rocha, Martín Sempere y Sebastián, 2008). Asimismo, como lo asevera Gibbons (1998):

En lo que a las universidades se refiere, la modificación más profunda es que la producción y la divulgación del conocimiento la investigación y la enseñanza ya no son actividades autónomas que se llevan a cabo en instituciones relativamente aisladas. Ahora implican una interacción con otros diversos productores de conocimiento.

Con respecto a esta estructura, el Sistema de Investigación Científica y Tecnológica de la Universidad Nacional Autónoma de Honduras (UNAH) está conformado por órganos gestores de la investigación: unidades de gestión de la investigación y coordinaciones regionales de investigación y órganos ejecutores de la actividad científica: institutos de investigación, observatorios universitarios y grupos de investigación:

La política de investigación de la UNAH persigue el fomento de la actividad científica y tecnológica, impulsando la asociabilidad y colaboración entre investigadores de las diferentes instancias, lo que se materializa con mayor claridad a través del desarrollo y consolidación de grupos de trabajo especializados (Salomón y Macías, 2011).

Igualmente, sobre este punto, Gibbons (1994) afirma que:

\footnotetext{
${ }^{1}$ Dirección de Investigación Científica y Posgrado, Universidad Nacional Autónoma de Honduras. Contacto: ivingzelaya@gmail.com
} 
Dentro del discurso sobre el nuevo modo de producción de conocimiento se viene a afirmar que, frente a la organización jerárquica y funcional tradicional (facultades, departamentos, disciplinas), se impondría el equipo de proyecto multidisciplinar, distribuido, flexible y temporal como forma de producción de conocimiento aplicado.

Así, desde el año 2013, la composición del Sistema de Investigación Científica y Tecnológica de la UNAH ha mostrado una tendencia creciente hacia la conformación de los grupos de investigación y un mayor dinamismo de estos en el desarrollo de actividades.

Aunque en la UNAH han funcionado, históricamente, algunos equipos colaborativos que siguiendo la definición de Reeves (1971) podrían considerarse grupos de investigación de facto, puesto que son "asociaciones de dos o más personas con objetivos comunes", fue hasta el año 2013 que la Dirección de Investigación Científica y Posgrado de la UNAH puso en marcha una estrategia explícita para fomentar el registro y consolidación de grupos de investigación estables, no vinculados al desarrollo de un proyecto o una iniciativa específica, sino, más bien, al desarrollo permanente de actividades en una temática determinada.

Estos grupos de investigación, aunque forman parte del Sistema de Investigación Científica y Tecnológica de la UNAH, fueron concebidos como: "Unidades organizativas de naturaleza funcional, pero con características estructurales dinámicas, frente a otras unidades como los departamentos, que muestran un carácter más estático" (Rey Rocha, Martín Sempere y Sebastián, 2008).

En tales estructuras plásticas que permiten el paso transitorio de estudiantes y colaboradores, como lo indica Etzkowitz (1992), su continuidad no está relacionada con la participación constante de los miembros a lo largo del tiempo, sino con el desarrollo de una línea de investigación estable y la transferencia de conocimientos tácitos y experiencias derivadas del trabajo conjunto a investigadores más noveles.

A finales del primer semestre de 2014, la UNAH contaba con 18 grupos de investigación activos. Estos grupos se encontraban conformados en torno a temáticas muy específicas que están insertas dentro de las prioridades de investigación definidas por la UNAH para el período 2012-2016.

En la tabla 1 se presenta la relación de grupos de investigación activos en la UNAH en el mes de julio de 2014, agrupados por facultades o centros a los que se encuentran adscritos. 
Tabla 1. Grupos de investigación activos a julio de 2014

\begin{tabular}{|c|c|}
\hline Facultad & Temática de investigación \\
\hline $\begin{array}{l}\text { Facultad de Ciencias } \\
\text { Médicas }\end{array}$ & $\begin{array}{l}\text { 1. Parasitismo intestinal y síndrome anémico } \\
\text { 2. Investigación en infecciones de etiología } \\
\text { bacteriana }\end{array}$ \\
\hline Facultad de Ciencias & $\begin{array}{l}\text { 3. Enfermedades virales humanas en Honduras } \\
\text { 4. Plantas útiles, taxonomía vegetal, cultivo de } \\
\text { hongos y ecología vegetal } \\
\text { 5. Parasitología }\end{array}$ \\
\hline $\begin{array}{l}\text { Facultad de Ciencias } \\
\text { Sociales }\end{array}$ & $\begin{array}{l}\text { 6. Pobreza, inequidad, cultura y desarrollo local } \\
\text { 7. Políticas públicas de infancia y juventud } \\
\text { 8. Desigualdad y exclusión social }\end{array}$ \\
\hline Facultad de Ingeniería & 9. Cambio climático y vulnerabilidad \\
\hline $\begin{array}{l}\text { Facultad de Química y } \\
\text { Farmacia }\end{array}$ & $\begin{array}{l}\text { 10. Estudio etnobotánico de la flora hondureña y } \\
\text { su potencial en salud y cambio climático } \\
\text { 11. Investigación fitoquímica } \\
\text { 12. Investigación en formulación farmacéutica de } \\
\text { productos fitoterapéuticos } \\
\text { 13. Investigación en análisis químicos en agua, } \\
\text { suelos, alimentos y medicamentos } \\
\text { 14. Productos naturales }\end{array}$ \\
\hline $\begin{array}{l}\text { Facultad de Ciencias } \\
\text { Espaciales }\end{array}$ & 15. Astronomía cultural \\
\hline $\begin{array}{l}\text { Facultad de } \\
\text { Humanidades }\end{array}$ & $\begin{array}{l}\text { 16. Investigación filológica de la literatura de } \\
\text { viajes escrita sobre Honduras entre los siglos } \\
\text { XIX y XX }\end{array}$ \\
\hline $\begin{array}{l}\text { Centro Universitario } \\
\text { Regional del Litoral } \\
\text { Pacífico }\end{array}$ & $\begin{array}{l}\text { 17. Desarrollo en la acuicultura y manejo de } \\
\text { recursos marino costeros }\end{array}$ \\
\hline $\begin{array}{l}\text { Centro Universitario } \\
\text { Regional del Litoral } \\
\text { Atlántico }\end{array}$ & 18. Ecosistemas acuáticos \\
\hline
\end{tabular}

Fuente: Elaboración propia a partir de los registros de la Dirección de investigación Científica de la UNAH, julio de 2014.

Estos grupos de investigación científica fueron conformados de manera voluntaria, con atención a ciertos requisitos mínimos en cuanto a su tamaño y estructura: están constituidos por al menos cuatro miembros que "realizan reflexión, debate, 
divulgación e investigación sobre un tema definido como prioritario por la UNAH" (Dirección de Investigación Científica, UNAH, 2013). Al menos un $50 \%$ de los miembros del grupo son profesores permanentes de la institución; los restantes pueden ser estudiantes de pregrado o posgrado y, en casos excepcionales, colaboradores profesionales externos a la Universidad que por su trayectoria en la línea de investigación definida prestan asistencia y trabajan de manera conjunta con el colectivo.

La asociación de investigadores en el grupo es dinámica y evoluciona en consonancia con las modificaciones internas de la unidad y las situaciones particulares de los miembros; así como por los cambios que se dan en las particularidades y demandas del contexto. Usualmente, la participación de estudiantes se produce de manera transitoria, en tanto que los investigadores de larga trayectoria normalmente adoptan un compromiso de larga duración con los proyectos.

Aunque las relaciones entre los miembros más experimentados de los grupos tienen una tendencia horizontal, existe cierta estructuración jerárquica: la función de liderazgo recae sobre un coordinador nombrado de manera consensuada, quien sirve de enlace con el resto de estamentos de gestión y ejecución de la estructura de investigación universitaria. Los grupos se encuentran adscritos a una Unidad de Gestión de la Investigación o Coordinación Regional de Investigación, las que avalan su labor y brindan apoyo y seguimiento a los procesos de fortalecimiento y ejecución de actividades planificados por el grupo.

De acuerdo al instructivo para constituir grupos de investigación científica en la UNAH (Dirección de Investigación Científica, 2013), las funciones que orientan el quehacer de los grupos de investigación científica son las siguientes:

1. Realizar de forma periódica reuniones de estudio y debate para discutir artículos, documentos científicos e institucionales, diagnósticos y demás relacionados con la temática de investigación definida.

2. Elaborar proyectos específicos que, dentro de la temática de investigación del grupo, compitan por fondos de la UNAH y entidades externas.

3. Organizar actividades de divulgación (conferencias, conversatorios, foros, simposios) con la participación de miembros de la comunidad universitaria y personas externas a la institución y participar con ponencias en Congresos científicos nacionales e internacionales.

4. Elaborar documentos de posicionamiento de la UNAH en torno a temas, procesos y decisiones relacionadas con el tema de investigación desarrollado. 
5. Elaborar artículos publicables para revistas nacionales e internacionales sobre las temáticas priorizadas por el grupo.

6. Establecer relaciones colaborativas con oficinas de Gobierno, cooperantes, universidades nacionales e internacionales, sector social y productivo para la transferencia de conocimiento y el desarrollo de proyectos conjuntos

7. Constituir la base de expertos temáticos de la UNAH, registrada y actualizada en la Dirección de Investigación Científica, con el propósito de participar en investigaciones, asesorar tesis, realizar consultorías, hacer revisiones de pares ydemás.

Estos lineamientos sirven de guía para la construcción de planes operativos bienales, que dependiendo de las particularidades y potencialidades del colectivo, incluyen actividades diversas con resultados y productos distintos, normalmente con un enfoque multi o transdiciplinario en el que prima la investigación aplicada y donde las acciones se plantean con relación a su contexto de aprovechamiento. Los grupos albergan en su seno actividades de investigación y divulgación, de transferencia de saberes y gestión de recursos e iniciativas de formación, capacitación y promoción científica.

En el primer año de funcionamiento de los grupos de investigación en la UNAH, la mayor parte de las actividades se orientó al desarrollo de reuniones internas de estudio y debate, organización de eventos de actualización del conocimiento con expertos nacionales e internacionales y desarrollo de propuestas de proyectos de investigación que, generalmente, fueron presentadas al fondo competitivo interno de becas.

La experiencia adquirida en la gestión de iniciativas colectivas, junto a una mayor cohesión y comunicación entre los miembros estables del grupo, propicia una constante ampliación de los ámbitos de trabajo dentro de la temática seleccionada. Se espera que, de manera progresiva, se generen actividades de capacitación, publicaciones conjuntas entre los miembros del grupo, contribuciones a congresos científicos, documentos que establezcan los posicionamientos de los académicos de la UNAH en torno a sucesos y tópicos concretos, hasta llegar a la realización de acciones de vinculación para la transferencia de saberes y tecnologías y la prestación de servicios.

Como se mencionó anteriormente, uno de los aportes fundamentales de los grupos de investigación para la institucionalización de la actividad científica en la educación superior consiste en la formación de investigadores y la transferencia de conocimientos para el relevo científico. Tanto los profesores universitarios con 
menos experiencia como los estudiantes que participan en el desarrollo de las actividades programadas, ven fortalecidas sus competencias investigativas en el marco de la temática específica del grupo. El trabajo estable y constante y la interacción entre los miembros del colectivo ayuda a garantizar una orientación teórica y metodológica conducida por los investigadores de larga trayectoria y que será clave para garantizar la conservación de las capacidades instaladas en la UNAH.

Adicionalmente, la existencia de grupos de investigación afines a carreras de posgrado constituye una clara fuente de docentes capacitados para realizar asesorías temáticas y metodológicas de trabajos de tesis, insertos en sus líneas de investigación. En tal sentido, se abren vías de colaboración entre los grupos y las carreras que posibilitan el desarrollo de investigaciones temáticas de mayor alcance, lideradas por los grupos y en las cuales los estudiantes de posgrado pueden forjar sus trabajos de graduación en el marco de procesos más amplios y con mayor repercusión en el ámbito de país.

Los grupos de investigación se constituyen en nexos ideales para propiciar e integrar redes académicas que faciliten la transferencia de conocimientos a la sociedad, como las caracterizadas por Gutiérrez (2006):

Con fundamento en la producción de flujos de conocimiento desde el contexto de las instituciones de educación superior y su articulación con las dimensiones organizacionales y funcionales del sector productivo y el Estado, dinamizando entre los agentes que los conforman, interrelaciones sistémicas que favorezcan la gestión del desarrollo.

En un escenario global, también los grupos de investigación pueden acoplarse con mayor facilidad a los estamentos de cooperación científica internacional. No obstante, una tarea pendiente para los gestores de investigación de la UNAH consiste en la construcción de una estrategia definida para la articulación de la investigación realizada por los grupos consolidados a procesos de innovación vinculados a distintos actores sociales. Del mismo modo, la incipiente dinámica y cultura de la innovación a lo interno de la Universidad y la falta de conceptos y protocolos claros que faciliten la interlocución e intercambio con agentes externos, son obstáculos que será necesario superar para el desarrollo de capacidades de gestión e investigación que fomenten la integración del conocimiento generado con su contexto de aplicación.

Como lo refiere Sebastián (2000): "La extensión de la cultura de la cooperación en la $|+D+|$ requiere de estrategias explícitas, expresadas a través de objetivos e 
instrumentos específicos en las políticas científicas, tecnológicas y de fomento de la innovación". En la UNAH, la Dirección de Investigación Científica, como órgano ejecutivo de la política de investigación, realiza esfuerzos sistemáticos para apoyar la conformación y fomentar las acciones de fortalecimiento de los grupos de investigación.

Varios autores aseveran que uno de los factores de gran incidencia en la consecución de resultados relevantes por los grupos es la clara definición de sus líneas de investigación. En el caso de la UNAH, el propio grupo determina su temática de investigación desde una óptica operativa, dentro de un marco de prioridades establecido quinquenalmente por la institución. La construcción de una estrategia de trabajo a dos años, liderada por el coordinador, que contemple propuestas de proyectos concretos concebidos en estrecha relación con las capacidades instaladas y potencialidad para la gestión de recursos por parte del grupo, es una condición para el registro oficial de los grupos.

Sumado a esto, la estrategia implementada focaliza la motivación y el reconocimiento a los componentes de los grupos de investigación por el desarrollo de actividades científicas y la generación de productos tangibles. Una de las prácticas desarrolladas por la UNAH, en este sentido, es la publicación de un Catálogo de grupos de investigación, en el cual los asociados puedan reconocer a sus pares y reconocerse a sí mismos como dinamizadores de una: "Cultura de la cooperación en la |+D+l que se fundamenta en la necesidad de la complementación de las capacidades" (Sebastián, 2000).

Los mecanismos de asignación de fondos destinados a la l+D+i dentro del Sistema de Investigación Científica y Tecnológica de la UNAH priorizan las propuestas presentadas por los grupos de investigación consolidados. Se conceden facilidades y medios de financiamiento específicos para la organización de encuentros académicos y la producción de materiales de divulgación científica; igualmente se da prioridad al otorgamiento de becas de investigación y acompañamiento para la elaboración de proyectos y presentación de propuestas para el financiamiento por otras entidades.

Como lo señalan Cuadros, Martínez y Torres (2008), la tarea de la Universidad consiste en facilitar mecanismos apropiados para que su entorno socioeconómico use y aproveche los conocimientos y resultados obtenidos por los grupos de investigación.

Los órganos de gestión de la investigación realizan una labor de monitoreo permanente y sistematizan la relación de actividades desarrolladas y productos 
generados por el grupo puesto que, en cuanto a la gestión de recursos externos, se ha demostrado que: "La interdisciplinariedad, los años de existencia del grupo y la producción previa influyen positivamente en la aprobación de proyectos a grupos de investigación en convocatorias internacionales" (Cuadros, Martínez y Torres, 2008).

A mediano plazo, contar con varios grupos de investigación trabajando en las diferentes aristas de un tema prioritario, podría dar pie a la conformación de unidades estructurales más complejas que aglutinen los esfuerzos desarrollados en el ámbito universitario. De esta manera, un conjunto de grupos de investigación que colaboran entre sí, pueden constituirse en un instituto de investigación que, con una mayor independencia administrativa, cumpla con:

Un rol protagónico fundamental que permite, por un lado, agrupar equipos interdisciplinarios y por otro facilita el desarrollo de la investigación aplicada al avance científico y tecnológico así como la formación de los recursos humanos de alta calidad, para desarrollar procesos y productos innovadores que ayuden a resolver problemas de interés nacional (Salomón y Macías, 2011).

En la UNAH, los grupos de investigación son actualmente una de las principales instancias de ejecución de la investigación y su importancia dentro de la estructura universitaria es creciente. Su flexibilidad y autonomía son propicias para el desarrollo de acciones colaborativas y la efectiva interlocución tanto con actores locales como con redes internacionales de la academia o de sectores estatales, productivos y sociales.

Las características y evolución de los grupos de investigación en la UNAH son consecuentes con el paradigma de investigación modalidad 2, promovido por Michael Gibbons (1998): "Consonantes con la promoción de estructuras más horizontales y dinámicas, más reflexivas, transdisciplinarias y heterogéneas, capaces de interactuar con otros productores del conocimiento para aprovechar los saberes de manera creativa y realizar investigación para la generación de conocimiento en el marco de su contexto de aplicación.

\section{Bibliografía}

Arrubia, J., Oquendo, S., Preciado, J., \& Ossa, J. (2001). Factores clave de éxito de los grupos y centros de investigación de excelencia y consolidados de la Universidad de Antioquia. Uni-Pluri/Versidad, 1 (1), 17-23.

Cuadros, A., Martínez, Á., \& Torres, F. (2008). Deterinantes del éxito en la participación de los grupos de investigación latinoamericanos en programas de cooperación científica internacional. Interciencia , 33(11), 821-827. 
Etzkowitz, H. (2003). Research groups as "quasi-firms": the invention of the entrepeneurial university. Research Policy, 32 (1), 109-121.

Dirección de Investigación Científica, UNAH. (2013). Dirección de Investigación Científica y Posgrados, UNAH. Recuperado de:

http://investigacionyposgrado.unah.edu.hn/sitios/investigacionyposgrado/estruc turalnvestigacion/instEjecucion/Paginas/GruposdelnvestigaciónCient\%C3\%ADf ica.aspx

Dirección de Investigación Científica, UNAH. (2012). Prioridades de Investigación UNAH, 2012-2016. Recuperado de: http://investigacionyposgrado.unah.edu.hn

Gutierrez, C., Carrera, M., Marín, F., Narváez, M., \& Pérez, C. (2006). Integración de redes académicas para la gestión y el desarrollo endógeno regional. Multiciencias, 6 (3), 257-263.

Gibbons, M. (1998). Higher education relevance in the 21rst century. World Bank. Washington D.C.: UNESCO.

Gibbons, M., Lomoges, C., Nowotny, H., Schwartzman, S., Scott, P., \& Throw, M. (1994). La nueva producción del conocimiento: La dinámica de la ciencia y la investigación en las sociedades contemporáneas. Barcelona: Ediciones Pomares-Corredor.

Rey Rocha, J., Martín Sempere, M., \& Sebastián, J. (2008). Estructura y dinámica de los grupos de investigación. ARBOR Ciencia, Pensamiento y Cultura, CLXXXIV (732), 743-757.

Reeves, E. (1971). La dinámica del comportamiento de grupos. México D.F.: Editota Técnica S.A.

Salomón, L., \& Macías, M. (2011). La importancia de una política de investigación en las instituciones de educación superior. VII Congreso Universitario Centroamericano. Managua: CSUCA.

Sebastián, J. (2000). La cultura de lacooperación en la I-D-i. Espacios. Revista venezolana de gestión tecnológica, 2 (21), 165-180. 